\title{
EFFECTIVE AREA CONTROL BY TAILORING THE CENTRAL CORE HOLE IN AIR-CORE CYLINDRICAL PHOTONIC CRYSTAL WAVEGUIDES
}

\author{
Jeong $\operatorname{Kim}^{1 *}$ \\ ${ }^{1}$ Department of Electric, Electronic and Communication Engineering Education, \\ Chungnam National University, Daejeon 34134, South Korea \\ 1jikimi@cnu.ac.kr
}

\begin{abstract}
Air-core photonic crystal waveguides with cylindrically periodic index variations are first proposed, thereby making low-index cores. The proposed aircore cylindrical photonic crystal waveguides (APCWs) possess numerous novel properties, including large or small effective areas. Based on two computational analyses of the finite-difference time-domain and finite difference method for accurate and cross-verified results, sizing the central core provides a decent way to control the effective area. Compared to the conventional step-index or dispersionshifted optical fibers with effective areas of $50-100 \mu \mathrm{m}^{2}$ in the $1.5-\mu \mathrm{m}$ wavelength range, increased effective area results are well obtained for the designed APCW with $r_{1}=2 \mu \mathrm{m}$ and seven layers as $199.4 \mu \mathrm{m}^{2}$ at the operation wavelength of $1.3 \mu \mathrm{m}$ and $201.2 \mu \mathrm{m}^{2}$ at $1.55 \mu \mathrm{m}$. It is also confirmed that the chromatic dispersion is very small, manageable, and moreover close to zero around the operating wavelength of $1.2 \mu \mathrm{m}$. Additionally, depending on the desired applications, investigations for reasonable and efficient designing of the APCWs with respect to superior effective core areas can lead to useful improvements related to lightwave communications.
\end{abstract}

Keywords - Effective area, Air core, Cylindrical photonic crystal waveguides, Optical components

\section{INTRODUCTION}

Current optical waveguides work as one of the widest bandwidth media for data transmission links, and also crucially operate as light-signal processing components in lightwave communication systems [1] [2]. Photonic crystal waveguides from very new advanced technology have attracted considerable attention in research communities during recent decades for the purpose of even more enhancing the performance of existing optical communications [1] [3] [4]. Basically, the photonic crystal waveguides (PCWs) can be constructed of a single material, which is a primary additive advantage, in contrast to all other conventional fiber-optic types, which are normally manufactured using two or more materials. Only by properly designing the PCW geometrical structures with simple material compositions, numerous significant characteristics, such as single mode operation, anomalous or near-zero group-velocity dispersion, polarization-maintaining, and large or small effective areas, can be obtained over the entire wavelength range of interest [5] [6]

Received: March 27, 2019

Reviewed: May 31, 2019

Accepted: June 3, 2019

* Corresponding Author 
[7]. Moreover, improved design by adding an air hole in the central core region, for air-core cylindrical photonic crystal waveguides (APCWs) in this research, may offer more opportunities for reliable and new fiber-optic components.

Since a great deal of interest has been focused on optical system elements with wider bandwidths and better performances, photonic crystal microstructures may have provided important solutions. Especially, the enlargement of the effective mode area can provide the advantages of reduced nonlinear effects combined with a high beam quality, thus doing a better job in coupling light from large focal spot devices [8] [9] [10]. Special ways only by delicately designing the optical microstructures can create new advanced optical applications.

In this paper, APCWs as distinctive variants of the PCW are first proposed, and design parameters are explained in order to investigate the influences of air-core size and cladding periodicity conditions. For exact computational analyses, the finite-difference time-domain (FDTD) technique is briefly described, considering employment during initial investigation, and then the rigorous full-vector finite difference method (FDM) is extensively utilized for the accurate calculation of light-field characteristics. Remarkable design of the APCW with enormously large effective area as well as reasonable guidance properties for device applications is addressed in consecutive order. This new type of computational design approach can be reasonably expected to be useful in manufacturing processes and for fabrications of a variety of optical waveguide components.

\section{FDTD AND FDM ALGORITHMS FOR APCW ANALYSES}

An optical lightwave field is an electromagnetic vector field in nature, and thus its propagation characteristics are governed by the physical laws of electrodynamic phenomena which are collectively referred to as Maxwell's equations [11] [12]. As researched in recent decades, light signal guiding of the PCW, which constitutes photonic bandgap structures caused by the periodic arrangement of identical air holes, is attributed to constructive interference effect, and the lightwave interaction in the core and cladding with photonic microstructures provides light confinement and hence guidance of the light field along the waveguide. Although the propagation properties of complicated structures like arbitrary APCWs cannot be evaluated easily using analytical methods, there are ways to solve electromagnetic problems computationally.

Figure 1(a) shows a proposed APCW with cylindrically periodic index variations. Here, a central air hole is located in the core region, forming a low-index core with a variable radius of $r_{1}$, surrounded by a clad region composed of alternating-index and equal-thickness rings. As shown in Fig. 1(a), da and dg in the refractive index profile for the proposed geometry denote thicknesses of air and pure-silica glass layers [13], respectively. The three-dimensional view of a regular PCW is also illustrated in Fig. 1(b).

In this research, two numerical approaches of FDTD and FDM are addressed, with regards for extension to the analysis of holey microstructure fibers with arbitrary air-hole geometries. Each of these approaches has certain advantages [12]. Adopting the FDTD technique, the continuous electromagnetic wave in a finite volume of numerical space is sampled at distinct locations in a spatial computation lattice and at equally spaced sampling intervals in time. The sampled data at the points can be used for numerical computations of allowed modes in a given waveguide without generating spurious mode solutions.

The FDTD approach is an efficient technique for computation of the propagation constants of guided modes. However, this method is not well suited for the evaluation of individual mode field patterns, because the generating source is an 
impulse function in the time domain covering an infinite spectrum and, as a result, the electromagnetic field solution is a superposition of all possible modes. In order to alleviate this problem related to the propagation constants available from the FDTD, individual electromagnetic mode patterns are obtained by securely using the FDM, which can easily and thoroughly provide individual mode field solutions.

Maxwell's equations, which summarize the laws of physical electromagnetism, are the basis of the FDTD algorithm that has gained considerable popularity during the past several decades [14]. This approach can provide robust solutions and readily accommodate complex-valued material properties. An arbitrary material object can be approximated by building up computational unit cells for which field component positions are disposed with the desired values of electric permittivity and magnetic permeability. Once the geometry of the 3-dimensional object is specified in the numerical simulation domain, a generating wave source condition is modeled somewhere in the region. Initially assuming that all fields within the calculation domain are identically zero, the incident wave is accordingly enforced to enter the numerical computation region.

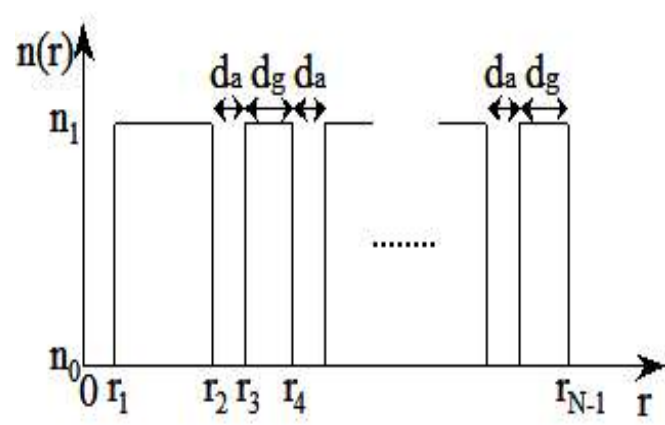

(a)

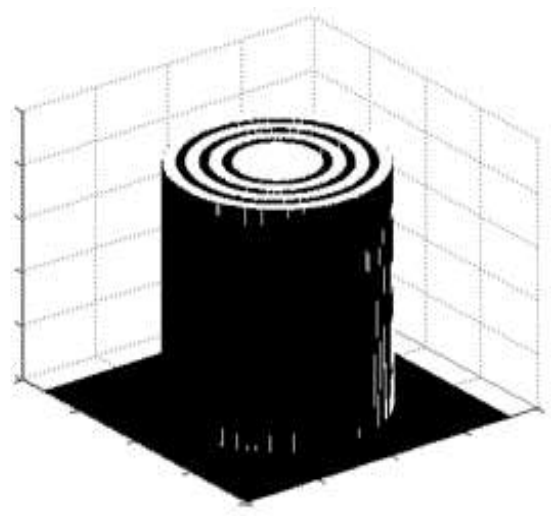

(b)

Fig. 1 Schematics of (a) the Refractive Index Profile of an APCW with an Air Core, and (b) by the Three-Dimensional View of a Regular PCW

Firstly, founding on the following two curl equations in the vectorial differential form among Maxwell's four expressions [11] [15]:

$$
\begin{aligned}
& \nabla \times \mathbf{E}=-\mu \frac{d \mathbf{H}}{d t} \\
& \nabla \times \mathbf{H}=\varepsilon \frac{d \mathbf{E}}{d t}
\end{aligned}
$$

where $\mu$ and $\varepsilon$ are the permeability constant and the permittivity constant, respectively, the system of six coupled partial differential equations are formulated for the FDTD analysis of electromagnetic wave interactions with general material objects. Due to the expanding of the curl expressions and equating the like components, the electric and magnetic field components (Ex, Ey, Ez, $\mathrm{Hx}, \mathrm{Hy}$, and $\mathrm{Hz}$ ) are interrelated. That is, Maxwell's equations do not directly produce electric and magnetic field values, but rather relate the rate of change between electric and magnetic field values.

Consequently, by applying logical and robust algorithms, complicated structures such as general PCWs and the proposed APCWs can be exploited to yield the propagation 
characteristics. Since cylindrically-varying PCWs have periodic index variations in the radial direction only, a high-index core PCW as in Fig. 1(b) with $\mathrm{r}_{1}=10 \mu \mathrm{m}$, da $=0.2 \mu \mathrm{m}, \mathrm{dg}=0.3 \mu \mathrm{m}$, and six layers is considered for the initial design. When assuming the glass portion of the designed waveguide has a refractive index of 1.45 with no material dispersion and operating the high-index core PCW at wavelength of $1.3 \mu \mathrm{m}$, the FDTD analysis produces the normalized propagation constant of 1.44923. Meanwhile, the FDM technique yields the effective refractive index of 1.44917 about the same operation wavelength. Only a negligible difference of $6 \times 10^{-5}$ is noticed. For the other low-index core APCW design with parameters of $\mathrm{r}_{1}$ $=0.3 \mu \mathrm{m}, \mathrm{r}_{2}=10 \mu \mathrm{m}, \mathrm{da}=0.2 \mu \mathrm{m}, \mathrm{dg}=0.3 \mu \mathrm{m}$, and seven layers, the effective index results from the FDTD and FDM techniques are 1.44465 and 1.44885, respectively, showing reasonable agreement as well.

\section{ELECTROMAGNETIC ANALYSIS RESULTS}

For the purpose of comparison of effective mode area variations in APCWs by tailoring the central air hole, another optimistic APCW is also designed with parameters of $\mathrm{r}_{1}=2 \mu \mathrm{m}, \mathrm{r}_{2}=10 \mu \mathrm{m}, \mathrm{da}=0.2 \mu \mathrm{m}, \mathrm{dg}=0.3 \mu \mathrm{m}$, and seven layers. Figure 2 illustrates the normalized propagation constant $(\bar{\beta})$ versus the operation wavelength $(\lambda)$ for the fundamental mode, obtained by the FDM approach taking into account the material dispersion effect [12].

In Fig. 2, it is noticed that three representative designs with and without the central air hole, aiming for the best effective area result, provide fairly reasonable propagation characteristics, because low-index air holes at the cores in the two APCW cases make the normalized propagation constant lowered in comparison with the 6-layer regular PCW. Here, the normalized propagation constant results for the APCWs with $\mathrm{r}_{1}=2.0 \mu \mathrm{m}$ and $\mathrm{r}_{1}=0.3 \mu \mathrm{m}$, and the 6-layer PCW are depicted by the green solid, red dotted, and blue dashed curves with the triangle symbol, respectively.

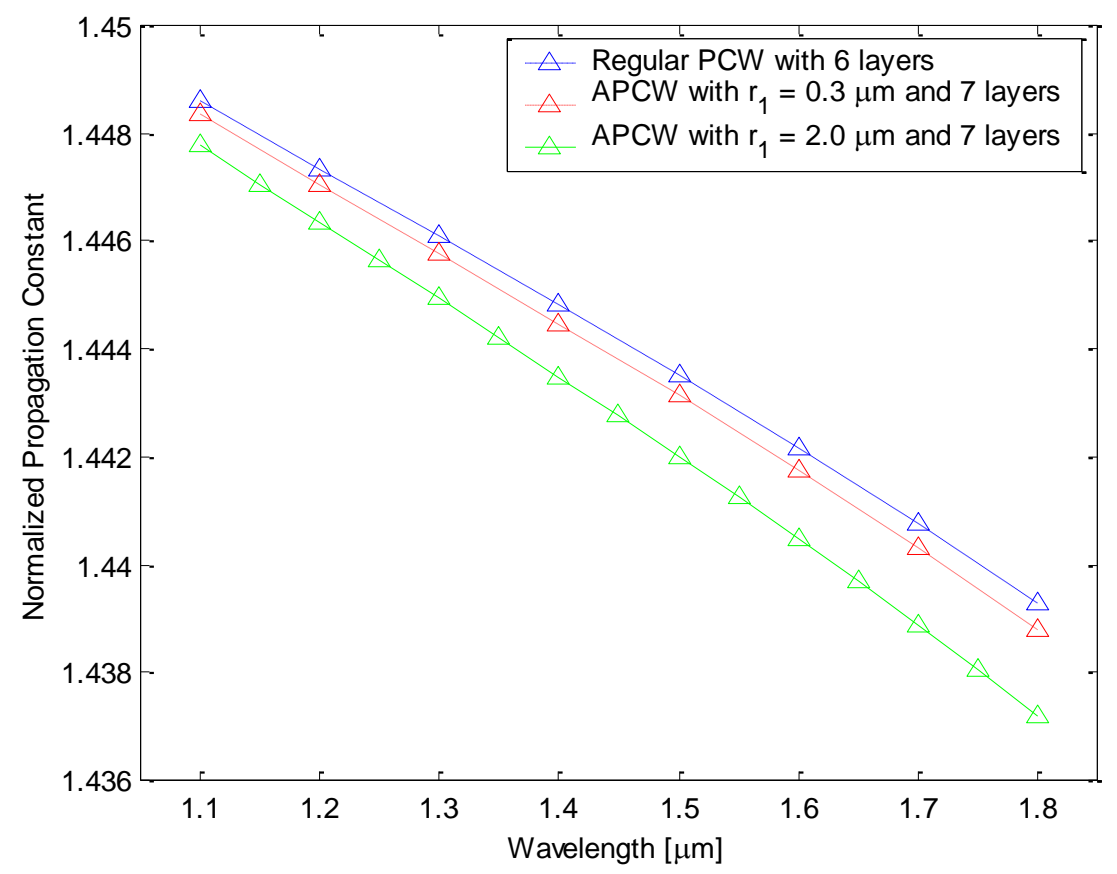


Fig. 2 Variations of the Normalized Propagation Constant Versus the Operation Wavelength

In order to check the dependency of electromagnetic characteristics on the number of outer layers, the normalized propagation constant results for two APCWs, as shown in Fig. 3, are examined. Figure 3(a) illustrates the refractive index profile for the APCW with $r_{1}=2 \mu \mathrm{m}$ and seven layers and Fig. 3(b) for the APCW with $r_{1}$ $=0.3 \mu \mathrm{m}$ and eleven layers. Here, the low-index layer of air is represented by the darker region and the pure fused-silica glass by the other region. The large or small central air hole in each design is clearly noticed.

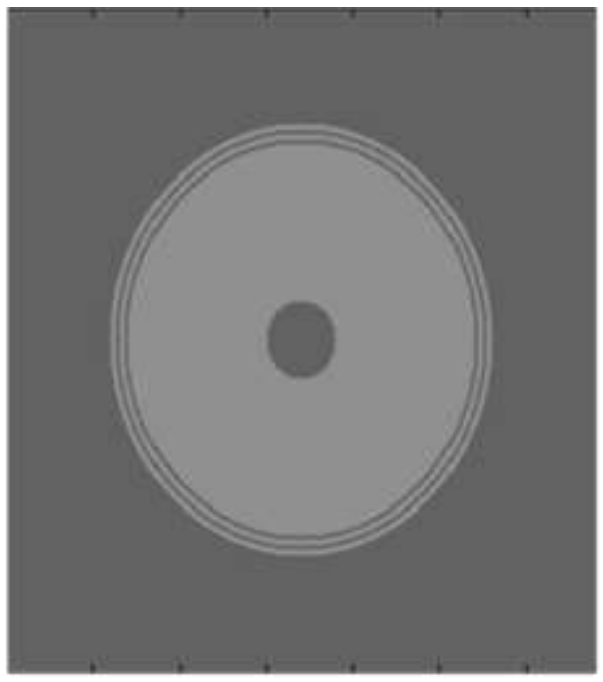

(a)

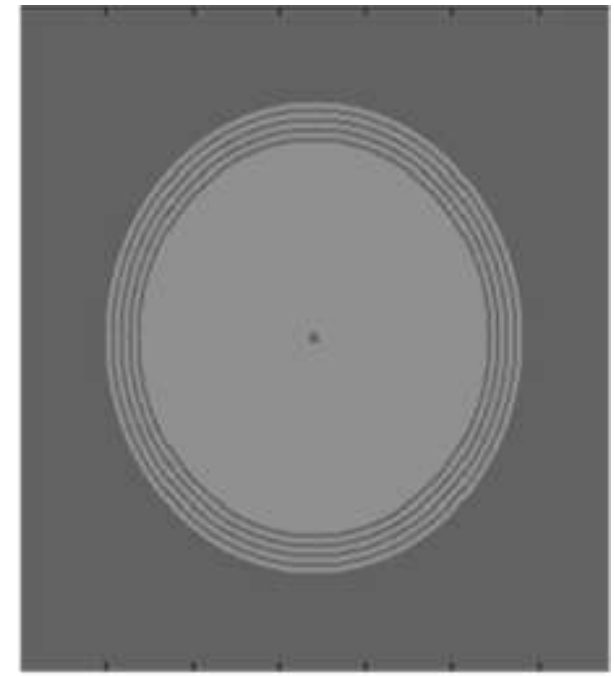

(b)

Fig. 3 Refractive Index Profiles of the APCWs with (a) $r_{1}=2 \mu \mathrm{m}$ and Seven Layers and (b) $r_{1}=0.3 \mu \mathrm{m}$ and Eleven Layers

Fig. 4 is an enlarged plot of normalized propagation constant variations by changing the number of outer layers from seven to eleven for the APCW with $r_{1}=$ $0.3 \mu \mathrm{m}$. Since the difference of the results is very small, the ordinate axis indicates the value obtained by subtracting 1.438 from the actual normalized propagation constant $(\bar{\beta})$. While the operation wavelength varies from 1.798 to $1.8 \mu \mathrm{m}$, the normalized propagation constant results for the nine- and eleven-layer APCWs with $\mathrm{r}_{1}=0.3 \mu \mathrm{m}$ are almost the same. The result for the seven-layer APCW, represented by the red dotted line, is about $0.387 \times 10^{-5}$ less than that for the nine-layer APCW at $\lambda=1.8 \mu \mathrm{m}$. An enlarged plot of $\bar{\beta}$ variations for the APCW case with $\mathrm{r}_{1}=2 \mu \mathrm{m}$ is also shown in Fig. 5. An even more negligible difference in results is noticed, and the curves appear to be almost overlapped. Hence, the APCWs with $r_{1}=0.3 \mu \mathrm{m}$ demonstrate more constant discrepancy than the waveguides with $r_{1}=2 \mu \mathrm{m}$ over the same wavelength range. It is reasonable that APCW with fewer layers provides lower normalized propagation constant overall. 


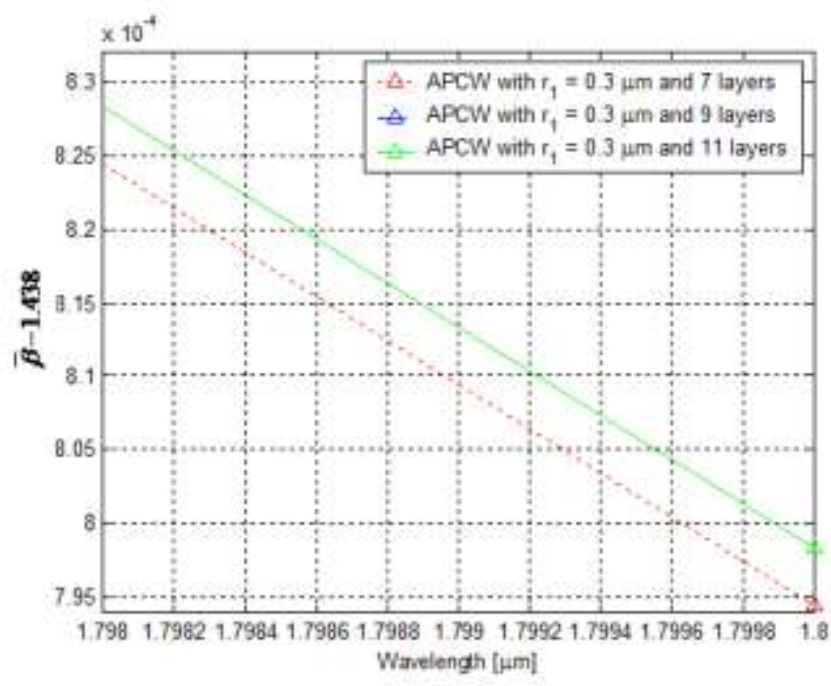

Fig. 4 Variations of the Normalized Propagation Constant versus the Operation Wavelength from 1.798 to $1.8 \mu \mathrm{m}$ by Changing the Number of Outer Layers of the APCW with $r_{1}=0.3 \mu \mathrm{m}$

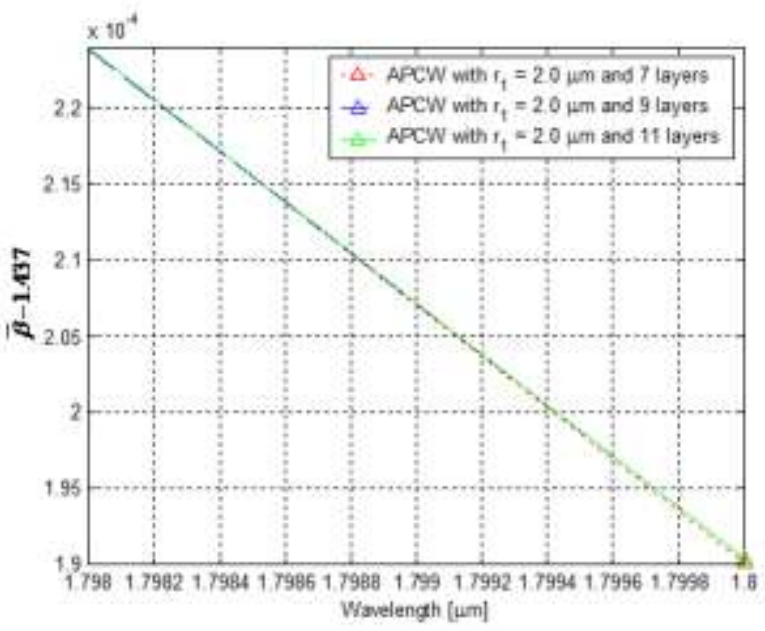

Fig. 5 Variations of the Normalized Propagation Constant Versus the Operation Wavelength from 1.798 to $1.8 \mu \mathrm{m}$ by Changing the Number of Outer Layers of the APCW with $\mathrm{r}_{1}=2.0 \mu \mathrm{m}$

Because it is verified that seven outer layers are enough for photonic crystal cladding in the proposed APCW, mode field distributions are investigated and then effective mode areas are evaluated by considering three PCW cases as in Fig 2. Only involving functions of spatial variables, the FDM technique is applied to produce electromagnetic field pattern quickly and conveniently. Figure 6 demonstrates the normalized Ex field distributions of the fundamental mode with the colorbar scale at the operating $\lambda=1.55 \mu \mathrm{m}$. 


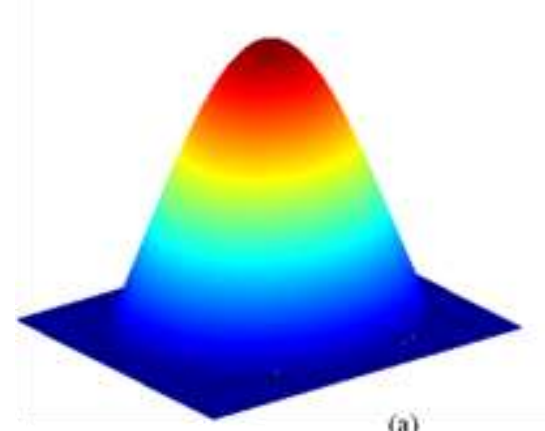

(a)

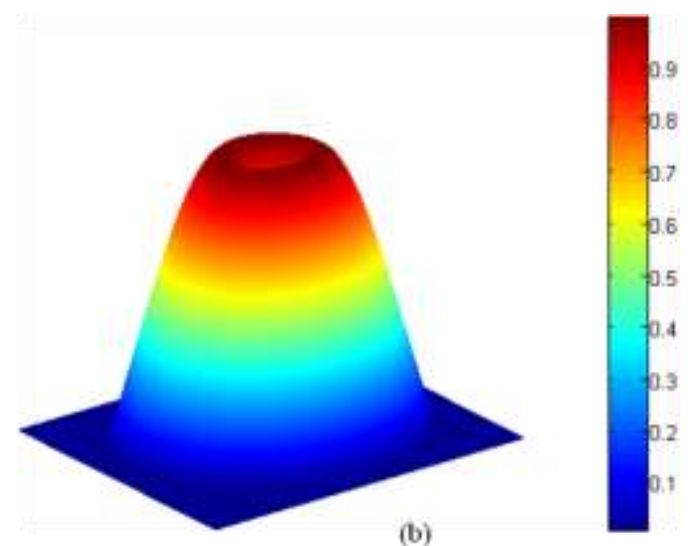

(b)

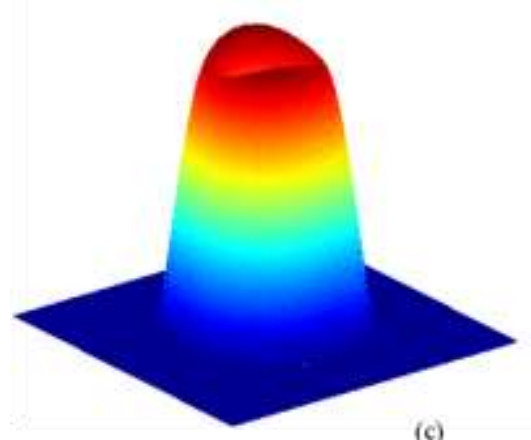

(c)
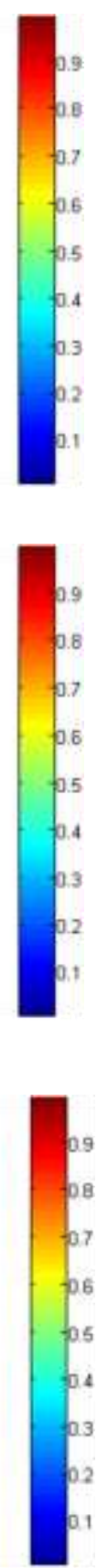

Fig. 6 Normalized Ex field distributions of the fundamental mode at the operating $\lambda=$ $1.55 \mu \mathrm{m}$ for (a) the regular PCW with six layers (b) the 7-layer APCW with $\mathrm{r}_{1}=0.3 \mu \mathrm{m}$ and (c) the 7-layer APCW with $\mathrm{r}_{1}=2 \mu \mathrm{m}$

The electric field component in Fig. 6(a) is obtained for the regular PCW case with six layers, by incorporating the material dispersion. The Ex components in Figs. 6(b) and 6(c) are produced for the 7-layer APCWs with $r_{1}=0.3 \mu \mathrm{m}$ and $r_{1}=2 \mu \mathrm{m}$, respectively. By increasing the size of the centre-air hole, a wider dip at the central region can be clearly observed, making more energy distributed cross the entire optical guiding region. This result also accords with the fact that electromagnetic fields tend to spread further in areas with higher refractive indices.

To better acknowledge the level of power spread concentration around optical guiding area, the effective areas of the fundamental mode are also calculated and 
compared for three cases, based on the electromagnetic field results, and plotted in Fig. 7. The effective area results for the APCWs with $r_{1}=2.0 \mu \mathrm{m}$ and $r_{1}=0.3 \mu \mathrm{m}$, and the 6-layer PCW are now denoted by the green solid, red dotted, and blue dashed curves with the diamond symbol, respectively. Since the mode fields spread more generally with longer operating wavelengths, the effective core areas for the 7-layer APCW with $\mathrm{r}_{1}=2.0 \mu \mathrm{m}$ and the regular 6-layer PCW at $\lambda=1.8 \mu \mathrm{m}$ are larger than at $\lambda=1.1 \mu \mathrm{m}$, although the area value for the 7-layer APCW with $\mathrm{r}_{1}=0.3 \mu \mathrm{m}$ is almost the same as $185 \times 10^{-12} \mathrm{~m}^{2}$ overall. As also clearly noticed, the 7-layer APCW with $\mathrm{r}_{1}=$ $2.0 \mu \mathrm{m}$ provides much larger effective areas (about $200 \mu \mathrm{m}^{2}$ ) than the other two cases, with differences of $15 \mu \mathrm{m}^{2}$ and $45 \mu \mathrm{m}^{2}$. This result reveals better performance, compared to the conventional step-index or dispersion-shifted optical fibers, which have effective areas of $50-100 \mu \mathrm{m}^{2}$ in the $1.5-\mu \mathrm{m}$ operation wavelength range [13]. Therefore, it is observed that the effective mode area tends to increase with an appropriately sized central hole, which is reasonable because lightwave fields spread by larger concentrations into the high-index cladding region. Thus tailoring the core air hole provides a decent design method to control the effective mode area.

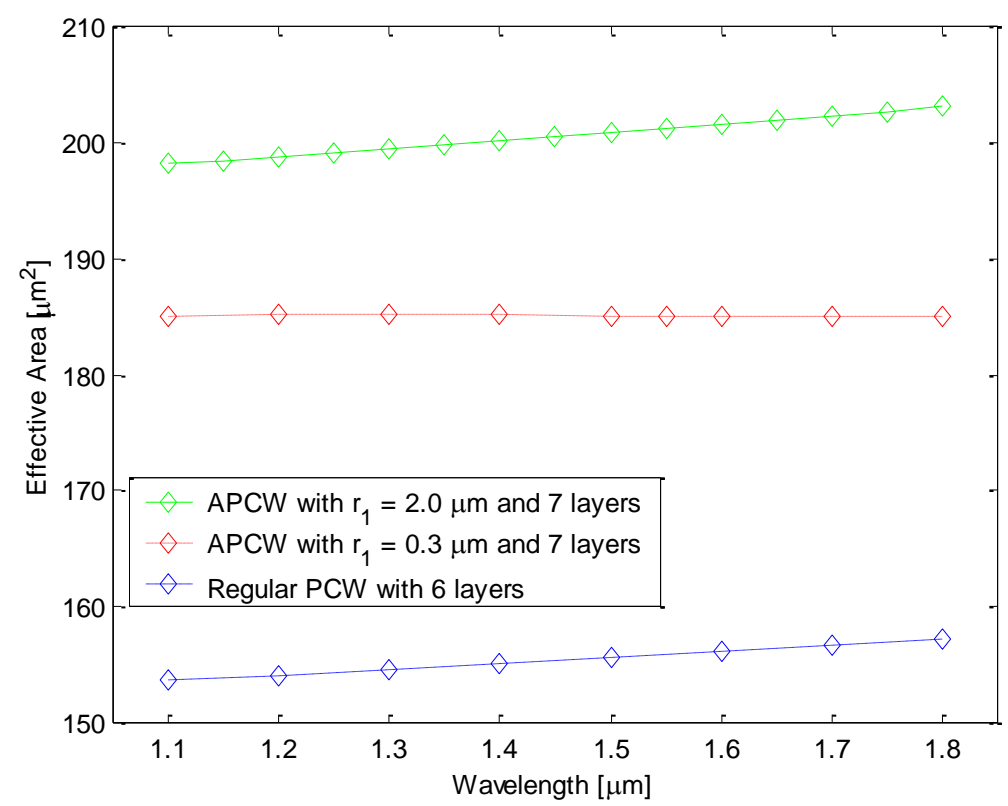

Fig. 7 Variations of the Effective Mode Area Versus the Operation Wavelength

Based on the FDM approach, further examination for the dispersion characteristic of the APCW with $r_{1}=2 \mu \mathrm{m}$ and seven layers is performed, accounting for the material dispersion. By using the normalized propagation constant result, the following second derivative equation is employed and evaluated for the chromatic dispersion [15]:

$$
D_{\text {chrom }}=-\frac{\lambda}{c} \frac{d^{2} \bar{\beta}}{d \lambda^{2}}
$$

where $\mathrm{c}$ is the speed of light in free space. Figure 8 illustrates the chromatic dispersion versus operating wavelength for the seven-layer APCW with $\mathrm{r}_{1}=2 \mu \mathrm{m}$, which provides superior effective mode area result. As observed, it is confirmed that the dispersion 
is very small and manageable and moreover close to zero around the operating condition of $\lambda=1.2 \mu \mathrm{m}$.

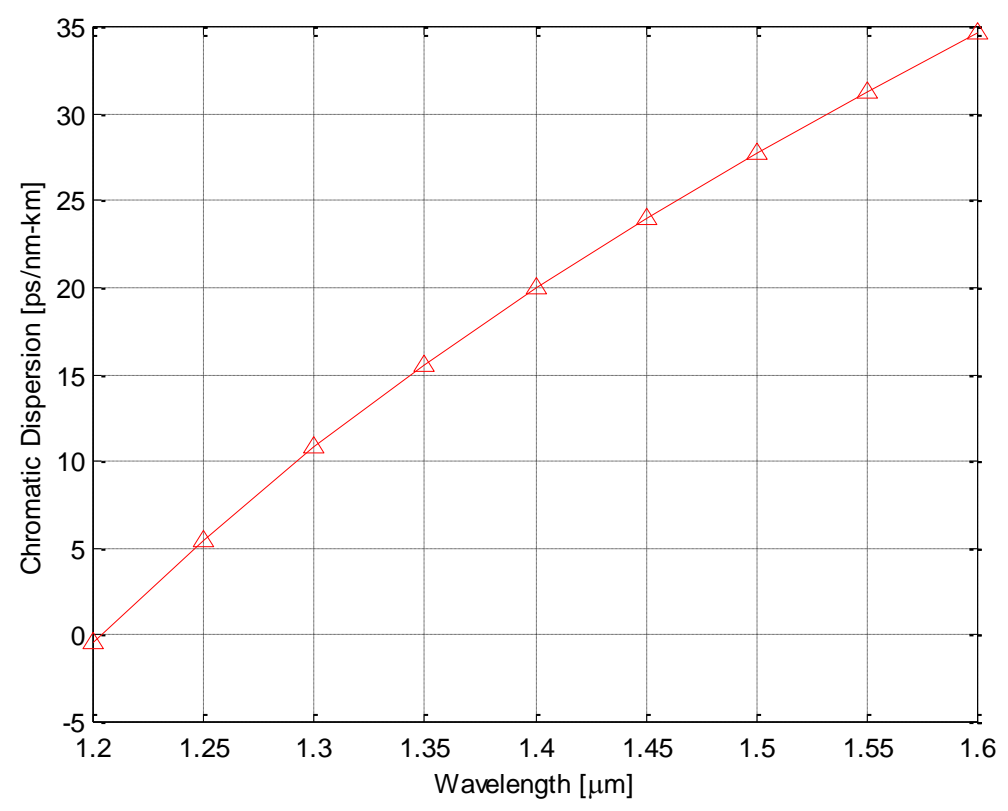

Fig. 8 Chromatic dispersion versus the operation wavelength for the fundamental mode in the seven-layer APCW with $\mathrm{r}_{1}=2 \mu \mathrm{m}$

\section{CONCLUSIONS}

Based on two computational approaches of the FDTD and FDM for accurate and cross-verified results, the proposed APCWs with cylindrically periodic index variations are designed and analyzed. Through intensive investigation, it is noticed that the effective mode area tends to increase with an appropriately sized central hole, which is reasonable because electromagnetic fields spread by larger concentrations into the high-index cladding region. This result conforms to the fact that generally as the operation wavelength becomes longer, fields spread further into the cladding region and the index-variation structure of the cladding part has more influence on the optical guidance properties, whereas lightwave fields are more confined to the central core with a major impact at the shorter wavelength. Increased effective area results are well obtained for the designed APCW with $\mathrm{r}_{1}=2 \mu \mathrm{m}$ and seven layers as $199.4 \mu \mathrm{m}^{2}$ at the operation wavelength of $1.3 \mu \mathrm{m}$ and $201.2 \mu \mathrm{m}^{2}$ at $1.55 \mu \mathrm{m}$.

Even though the proposed APCWs have complicated geometries, which require a full vectorial approach instead of a rather easy scalar-wave approximation and make the electromagnetic analysis difficult, the results in this research can be utilized as a general guide for the purpose of efficiently designing for a superior effective core area. Additionally, depending on the desired applications, investigations for reasonably designing of the APCWs with different parameters are expected to improve lightwave-related communication systems.

\section{ACKNOWLEDGMENTS}

The author would like to acknowledge the support of a financial grant from the CNU study program (18-0989-01) of Chungnam National University. 


\section{REFERENCES}

[1] M. Sharma and S. Konar, "Broadband Supercontinuum Generation in Lead Silicate Photonic Crystal Fibers Employing Optical Pulses of 50W Peak Power." Optics Communications 300 (2016): 310-319.

[2] M. Pourmand, A. Karimkhani, and M. K. Moravvej-Farshi. "Slow Light Photonic Crystal Waveguides with Large Delay-Bandwidth Product." Optical Engineering 55.12 (2016): 123108.

[3] E. K. Akowuah, T. Gorman, H. Ademgil, S. Haxha, G. K. Robinson, and J. V. Oliver. "Numerical Analysis of a Photonic Crystal Fiber for Biosensing Applications." IEEE Journal of Quantum Electronics 48.11 (2012): 1403-1410.

[4] S. Kalra, S. Vyas, M. Tiwari, O. Buryy, and G. Singh. "Highly Nonlinear Multi-Material Chalcogenide Spiral Photonic Crystal Fiber for Supercontinuum Generation." Acta Physica Polonica A 133.4 (2018): 1000-1002.

[5] J. Kim. "Design of Nonlinear Photonic Crystal Fibers with a Double-Cladded Coaxial Core for Zero Chromatic Dispersion." Applied Optics 51.28 (2012): 6896-6899.

[6] M. R. Hasan, M. S. Anower, and M. I. Hasan. "Polarization Maintaining Highly Nonlinear Photonic Crystal Fiber with Closely Lying Two Zero Dispersion Wavelengths." Optical Engineering 55.5 (2016): 056107.

[7] H. Y. Fu, H. Y. Tam, L. Y. Shao, X. Dong, P. K. A. Wai, C. Lu, and S. K. Khijwania. "Pressure Sensor Realized with Polarization-Maintaining Photonic Crystal Fiber-Based Sagnac Interferometer." Applied Optics 47.15 (2008): 2835-2839.

[8] P. St. J. Russell. "Photonic-Crystal Fibers." Journal of Lightwave Technology 24.12 (2006): 4729-4749.

[9] H. Y. Fu, H. Y. Tam, L. Y. Shao, X. Dong, P. K. A. Wai, C. Lu, and S. K. Khijwania. "Photonic Crystal Fibers, Devices, and Applications." Frontiers of Optoelectronics 6.1 (2013): 3-24.

[10] J. Kim. "Effective Area Control by Varying the Air Core Size of Cylindrical Photonic Crystal Waveguides." 2019 1st Korea-China Joint Workshop on Human-centric Idea, Policy and Advancement, Daejeon, Korea, and Qingdao, China, 12-14 April 2019. Vol. 10., International Journal of Energy, Information and Communications, 2019, pp. 1-6.

[11] C. A. Balanis. Advanced Engineering Electromagnetics. New York: Wiley, 1989.

[12] J. Kim. "Effects of Core and Cladding on Optical Guidance Properties of Holey Fibers". Optical Communication, InTech, 2012, pp. 25-40.

[13] G. P. Agrawal. Nonlinear Fiber Optics. San Diego: Academic Press, 2001.

[14] A. Taflove and S. C. Hagness. Computational Electrodynamics: the Finite-Difference Time-Domain Method. Boston: Artech House, 2005.

[15] G. Keiser. Optical Fiber Communications. New York: McGraw Hill, 2000. 